SUPPORTING INFORMATION FOR:

\title{
Zinc-Catalyzed Hydroalkoxylation/Cyclization of Alkynyl Alcohols
}

Felipe de la Cruz-Martínez, ${ }^{a}$ Marc Martínez de Sarasa Buchaca, ${ }^{a}$ Juan Fernández-Baeza, ${ }^{a}$ Luis F. Sánchez-Barba, ${ }^{b}$ Ana M. Rodríguez, ${ }^{a}$ José A. Castro-Osma, ${ }^{*, c}$ Agustín Lara-Sánchez *,a

aUniversidad de Castilla-La Mancha, Departamento de Química Inorgánica, Orgánica y BioquímicaCentro de Innovación en Química Avanzada (ORFEO-CINQA), Facultad de Ciencias y Tecnologías Químicas, 13071-Ciudad Real, Spain. E-mail: Agustin.Lara@uclm.es.

bDepartamento de Biología y Geología, Física y Química Inorgánica, Universidad Rey Juan Carlos, Móstoles, 28933 Madrid, Spain.

cUniversidad de Castilla-La Mancha, Departamento de Química Inorgánica, Orgánica y BioquímicaCentro de Innovación en Química Avanzada (ORFEO-CINQA), Facultad de Farmacia, 02071Albacete, Spain. E-mail: JoseAntonio.Castro@uclm.es. 


\section{Table of Contents}

$\begin{array}{ll}\text { 1. Experimental section } & \text { S3 }\end{array}$

1.1. General procedure for catalytic intramolecular hydroalkoxylation $\quad$ S3

1.2. General procedure for kinetic measurements $\quad$ S3

2. Figures and Tables referred to in main text $\quad$ S5

Figure S1. NMR spectra for [ZnMe $\left(\kappa^{3}\right.$-bpzbdmape $\left.)\right](\mathbf{1})$ in $\mathrm{C}_{6} \mathrm{D}_{6} \quad$ S5

Figure S2. NMR spectra for [ $\mathrm{ZnEt}\left(\kappa^{3}\right.$-bpzbdmape $\left.)\right](2)$ in $\mathrm{C}_{6} \mathrm{D}_{6} \quad \mathrm{~S} 6$

Figure S3. NMR spectra for $\left[\mathrm{Zn}\left\{\mathrm{N}\left(\mathrm{SiMe}_{3}\right)_{2}\right\}\left(\kappa^{3}\right.\right.$-bpzbdmape $\left.)\right](3)$ in $\mathrm{C}_{6} \mathrm{D}_{6} \quad \mathrm{~S} 7$

Figure S4. NMR spectra for $\left[\mathrm{Zn}\left\{\mathrm{N}\left(\mathrm{SiHMe}_{2}\right)_{2}\right\}\left(\kappa^{3}\right.\right.$-bpzbdmape $\left.)\right](4)$ in $_{6} \mathrm{C}_{6} \mathrm{D}_{6} \quad \mathrm{~S} 8$

Figure S5. NMR spectra for $\left[\mathrm{ZnMe}\left(\kappa^{3}\right.\right.$-bpzbdeape $\left.)\right](5)$ in $\mathrm{C}_{6} \mathrm{D}_{6} \quad \mathrm{~S} 9$

Figure S6. NMR spectra for [ZnEt $\left(\kappa^{3}\right.$-bpzbdeape) $)(6)$ in $\mathrm{C}_{6} \mathrm{D}_{6} \quad \mathrm{~S} 10$

Figure S7. NMR spectra for $\left[\mathrm{Zn}\left\{\mathrm{N}\left(\mathrm{SiMe}_{3}\right)_{2}\right\}\left(\kappa^{3}\right.\right.$-bpzbdeape $\left.)\right](7)$ in $\mathrm{C}_{6} \mathrm{D}_{6} \quad \mathrm{~S} 11$

Figure S8. NMR spectra for $\left[\mathrm{Zn}\left\{\mathrm{N}\left(\mathrm{SiHMe}_{2}\right)_{2}\right\}\left(\kappa^{3}\right.\right.$-bpzbdeape $\left.)\right](\mathbf{8})$ in $\mathrm{C}_{6} \mathrm{D}_{6} \quad \mathrm{~S} 12$

Figure S9. NMR spectra for $\left[\mathrm{Zn}\left\{\mathrm{C} \equiv \mathrm{C}\left(\mathrm{C}_{6} \mathrm{H}_{4}\right) \mathrm{CH}_{2} \mathrm{OH}\right\}\left(\kappa^{3}\right.\right.$-bpzbdmape $\left.)\right](\mathbf{1 5})$ in $\mathrm{CD}_{2} \mathrm{Cl}_{2} \quad \mathrm{~S} 13$

Figure S10. ${ }^{1} \mathrm{H}$ NMR monitoring of the hydroalkoxylation of 9 to $\mathbf{1 0}$ mediated by catalyst 8 in toluene- $d^{8}$ at $90^{\circ} \mathrm{C}$

Figure S11. Plot of [9] versus reaction time for the hydroalkoxylation of 9 catalyzed by catalyst 8 at different concentrations of $\mathbf{8}$

Figure S12. Arrhenius plot for the cyclization of 9 catalyzed by 8 over the temperature range $60-90$ ${ }^{\circ} \mathrm{C}$ in toluene- $d^{8}$

Figure S13. Eyring plot for the cyclization of 9 catalyzed by 8 over the temperature range $60-90{ }^{\circ} \mathrm{C}$ in toluene- $d^{8}$

Table S1. Crystal data and structure refinement for compounds 2, 3 and 15

Table S2. Selected bond distances $(\AA)$ and angles $\left(^{\circ}\right)$ for compounds 2, 3 and 15 


\section{Experimental section}

\subsection{General procedure for catalytic intramolecular hydroalkoxylation}

In a typical small-scale experiment, in a glovebox, catalyst 1-8 and alkynyl alcohol 9, 11 or 13, were dissolved in toluene- $d_{8}$ and placed in a J. Young style NMR tube with a resealable Teflon valve. The tube was closed and placed in a oil bath that was preheated to the desired temperature. The reaction was monitored by ${ }^{1} \mathrm{H}$ NMR spectroscopy to determine the conversion.

Representative example: In the catalytic cyclization of (2-ethynylphenyl)methanol (9) with catalyst $\mathbf{8}, 0.066 \mathrm{~g}(0.5 \mathrm{mmol})$ of alkynyl alcohol 9 and $0.018 \mathrm{~g}(0.025 \mathrm{mmol})$ of catalyst 8 were dissolved in toluene- $d_{8}(0.6 \mathrm{~mL})$. The resultant solution was quickly transferred to a J. Young style NMR tube which was immediately placed into an oil bath that was preheated at $90{ }^{\circ} \mathrm{C}$. Single scan spectra were acquired automatically at different time intervals. The concentration of substrate and product at any given time were determined by integration of substrate and product resonances.<smiles>C=C1OCc2ccccc21</smiles>

1-Methylene-1, 3-dihydroisobenzofuran (10). Spectral data matched literature references. ${ }^{1}{ }^{1} \mathrm{H}$ NMR (500 MHz, $\left.\mathrm{C}_{6} \mathrm{D}_{6}\right): \delta 7.19(\mathrm{~m}, 1 \mathrm{H}), 6.93(\mathrm{~m}, 2 \mathrm{H}), 6.64(\mathrm{~m}, 1 \mathrm{H}), 4.78(\mathrm{~s}, 2 \mathrm{H}), 4.71(\mathrm{~s}, 1 \mathrm{H}), 4.54$ $(\mathrm{s}, 1 \mathrm{H})$.<smiles>C=C1CCCO1</smiles>

2-Methylene-tetrahydrofuran (12). Spectral data matched literature references. ${ }^{2}{ }^{1} \mathrm{H}$ NMR (500 $\left.\mathrm{MHz}, \mathrm{C}_{6} \mathrm{D}_{6}\right): \delta 4.5(\mathrm{~s}, 1 \mathrm{H}), 3.9(\mathrm{~s}, 1 \mathrm{H}), 3.7(\mathrm{t}, 2 \mathrm{H}), 2.2(\mathrm{t}, 2 \mathrm{H}), 1.5(\mathrm{~s}, 1 \mathrm{H})$.<smiles>C=C1CCCCO1</smiles>

2-Methylene-tetrahydropyran (14). Spectral data matched literature references. ${ }^{2}{ }^{1} \mathrm{H}$ NMR (500 $\left.\mathrm{MHz}, \mathrm{C}_{6} \mathrm{D}_{6}\right): \delta 4.75(\mathrm{~s}, 1 \mathrm{H}), 4.25(\mathrm{~s}, 1 \mathrm{H}), 3.5(\mathrm{~m}, 2 \mathrm{H}), 2.0(\mathrm{~m}, 2 \mathrm{H}), 1.5(\mathrm{~m}, 4 \mathrm{H})$ 


\subsection{General procedure for kinetic measurements}

In a glovebox, a J. Young style NMR tube was charged with of the catalyst $\mathbf{8}$ and the substrate $\mathbf{9}$ in toluene- $d_{8}$. The conversion was monitored by ${ }^{1} \mathrm{H}$ NMR spectroscopy by following the disappearance of the alkyne signals of the substrate.

Representative example: In the kinetic studies to determine the rate dependence on catalyst in the cyclization of (2-ethynylphenyl)methanol (9), $0.044 \mathrm{~g}(0.33 \mathrm{mmol})$ of substrate 9 and a $96 \mu \mathrm{L}$ of solution containing a known concentration of catalyst $8\left(0.2205 \mathrm{M}\right.$ in toluene- $\left.d_{8}\right)$, were placed in a glass vial. Then, toluene- $d_{8}$ was added in order to complete the total volume of $0.6 \mathrm{~mL}$ and the dissolved mixture was transferred to a J. Young style NMR tube. The tube was closed and placed into an $\mathrm{RMN}$ spectrometer that was preheated at $90^{\circ} \mathrm{C}$. The reaction was monitored at regular intervals (2 min) by ${ }^{1} \mathrm{H}$ NMR spectroscopy. 


\section{Figures and Tables referred to in main text}

Figure S1. NMR spectra for $\left[\mathrm{ZnMe}\left(\kappa^{3}\right.\right.$-bpzbdmape $\left.)\right](\mathbf{1})$ in $\mathrm{C}_{6} \mathrm{D}_{6}$.
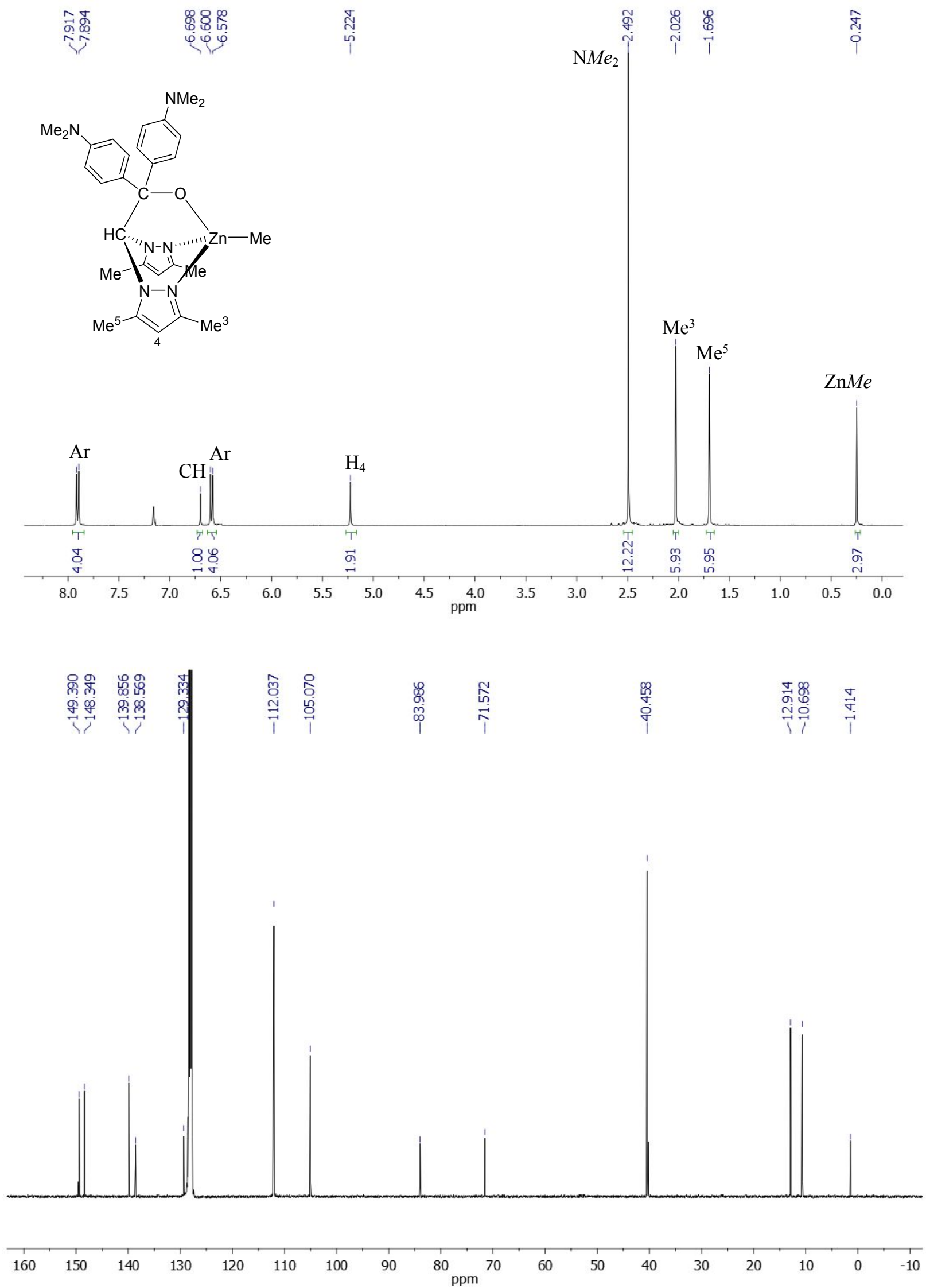
Figure S2. NMR spectra for $\left[\mathrm{ZnEt}\left(\kappa^{3}\right.\right.$-bpzbdmape $\left.)\right](2)$ in $\mathrm{C}_{6} \mathrm{D}_{6}$.
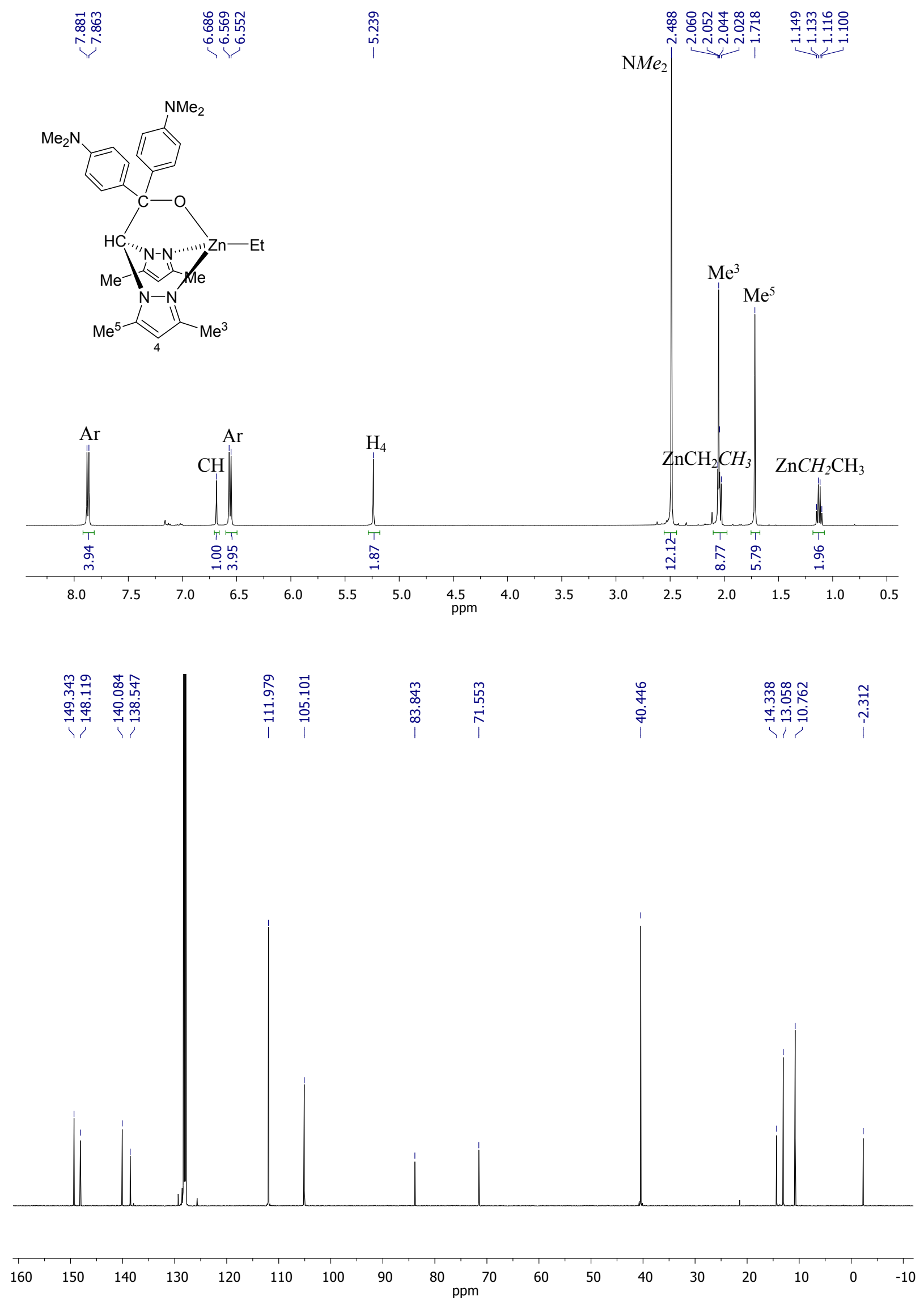
Figure S3. NMR spectra for $\left[\mathrm{Zn}\left\{\mathrm{N}\left(\mathrm{SiMe}_{3}\right)_{2}\right\}\left(\kappa^{3}\right.\right.$-bpzbdmape $\left.)\right](3)$ in $\mathrm{C}_{6} \mathrm{D}_{6}$.
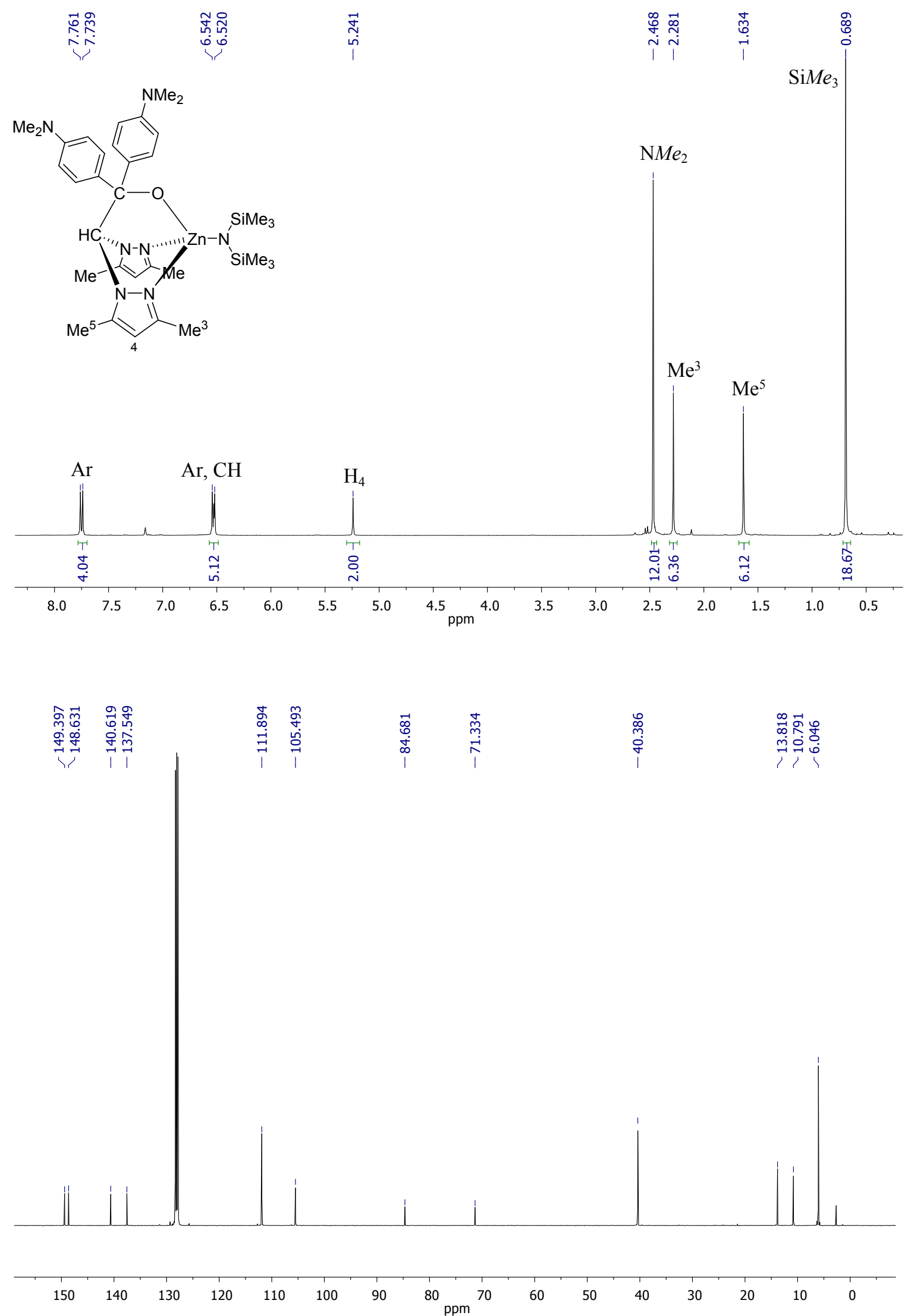
Figure S4. NMR spectra for $\left[\mathrm{Zn}\left\{\mathrm{N}\left(\mathrm{SiHMe}_{2}\right)_{2}\right\}\left(\kappa^{3}\right.\right.$-bpzbdmape $\left.)\right](4)$ in $\mathrm{C}_{6} \mathrm{D}_{6}$.
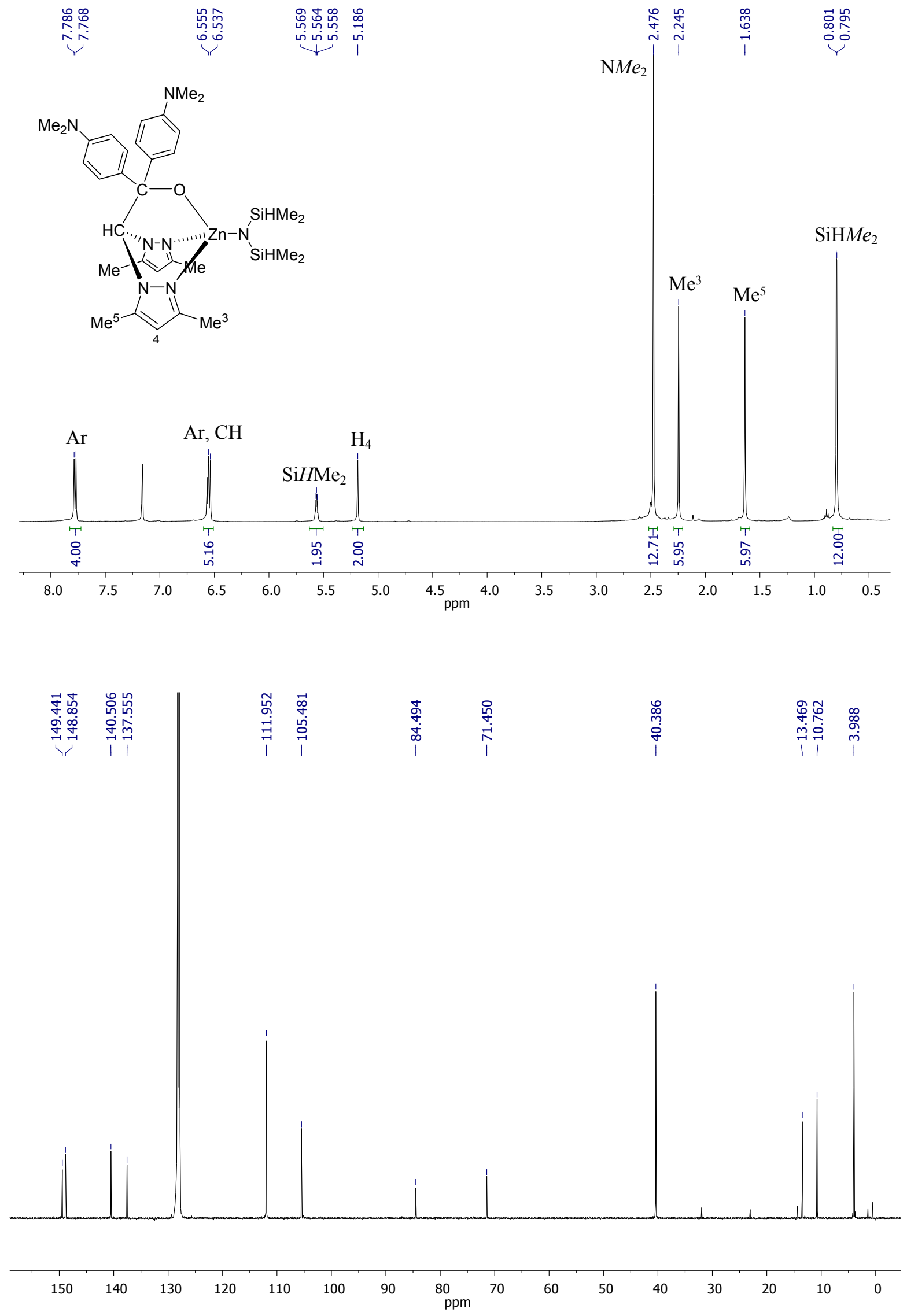
Figure S5. NMR spectra for $\left[\mathrm{ZnMe}\left(\kappa^{3}\right.\right.$-bpzbdeape $\left.)\right](5)$ in $\mathrm{C}_{6} \mathrm{D}_{6}$.
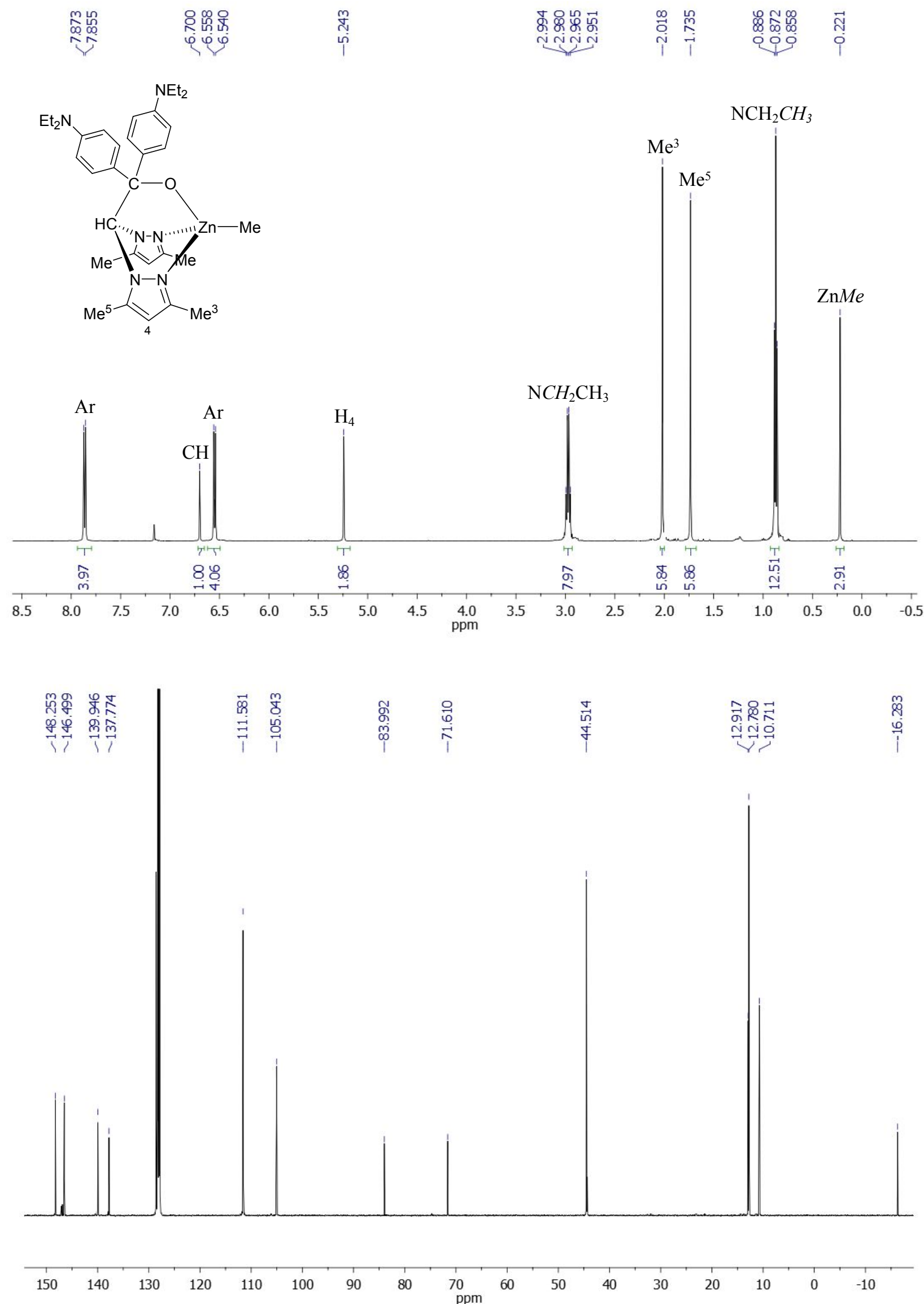
Figure S5. NMR spectra for $\left[\mathrm{ZnEt}\left(\kappa^{3}\right.\right.$-bpzbdeape $\left.)\right](6)$ in $\mathrm{C}_{6} \mathrm{D}_{6}$.
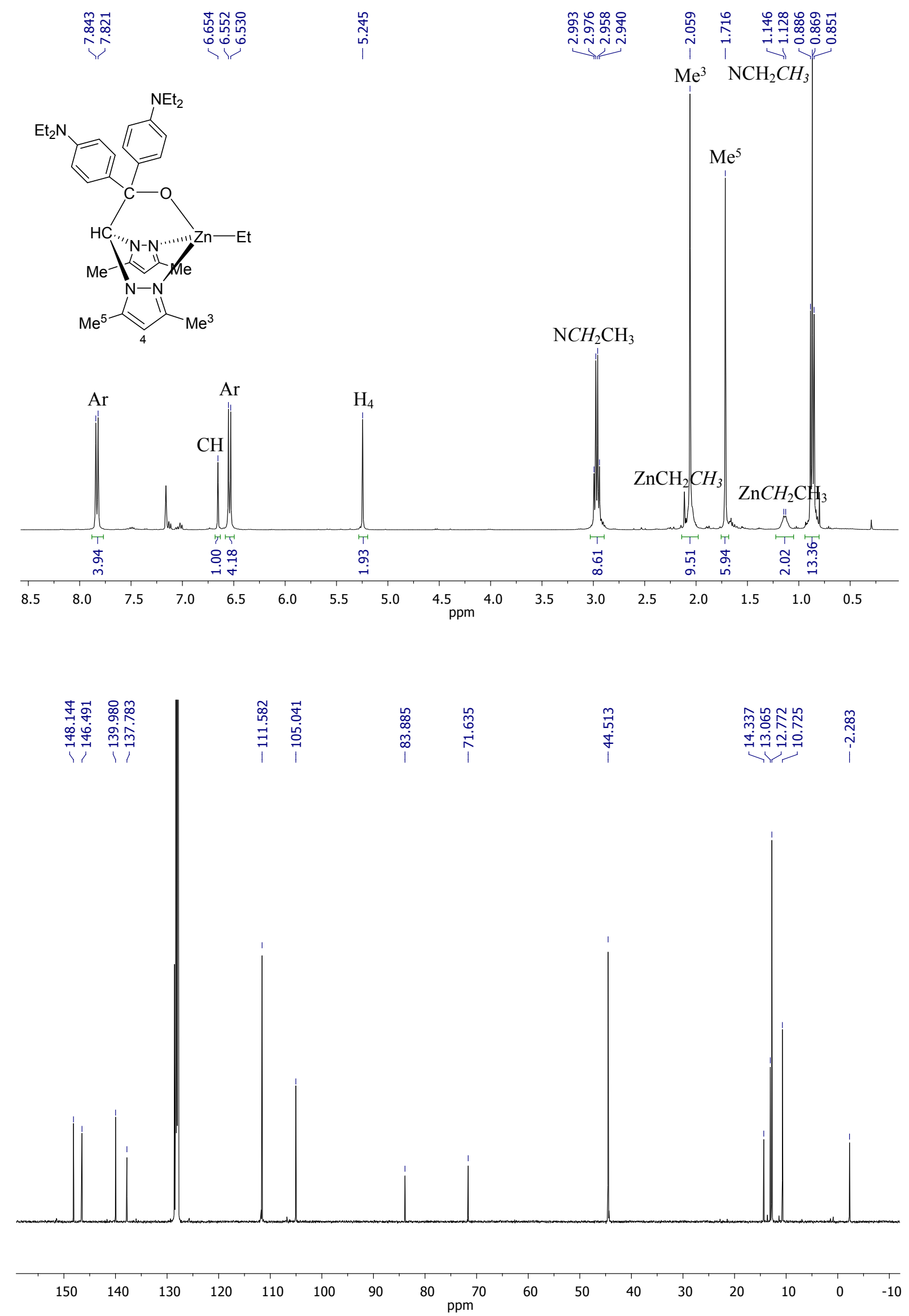
Figure S7. NMR spectra for $\left[\mathrm{Zn}\left\{\mathrm{N}\left(\mathrm{SiMe}_{3}\right)_{2}\right\}\left(\kappa^{3}\right.\right.$-bpzbdeape $\left.)\right](7)$ in $\mathrm{C}_{6} \mathrm{D}_{6}$.
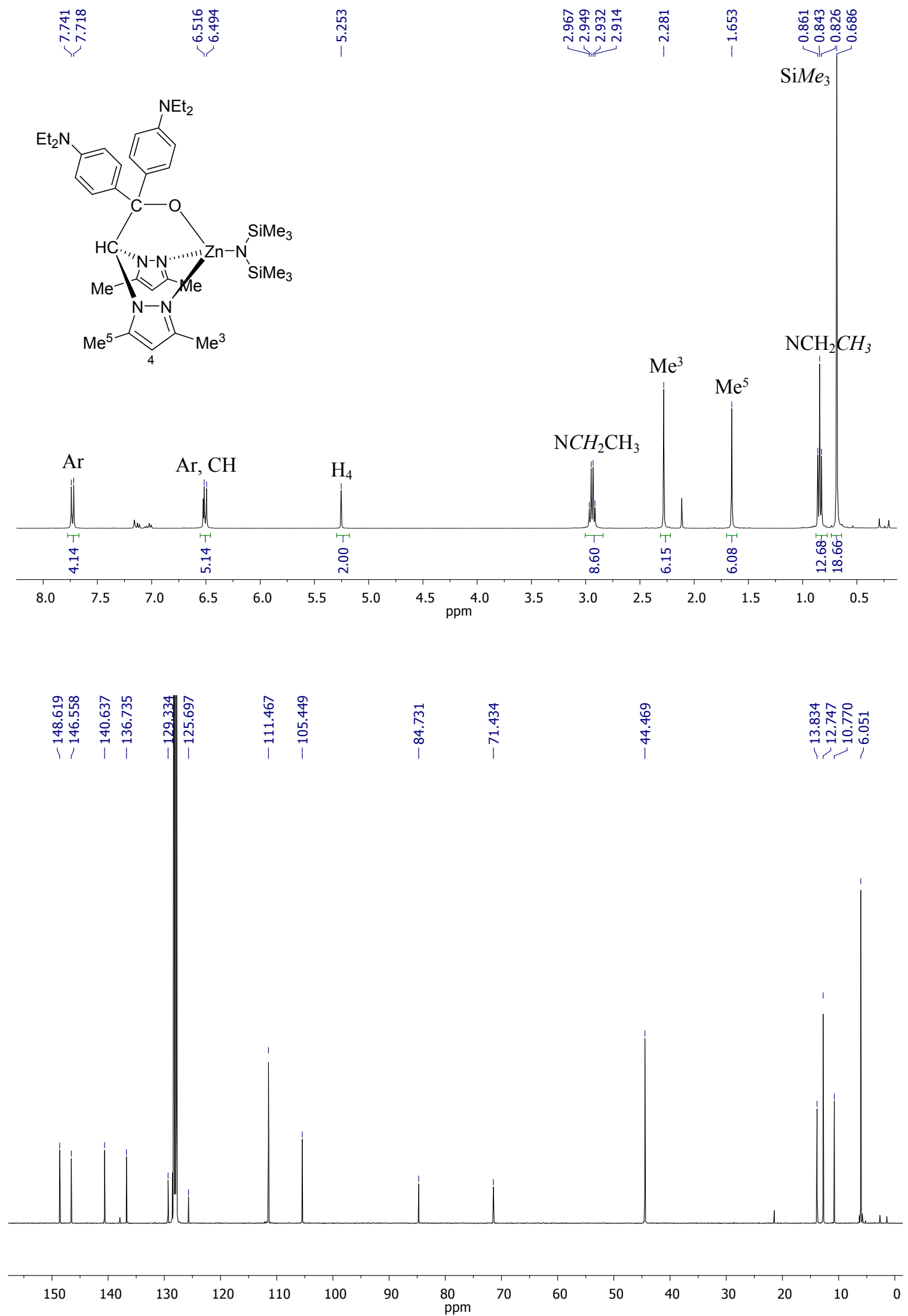
Figure S8. NMR spectra for $\left[\mathrm{Zn}\left\{\mathrm{N}\left(\mathrm{SiHMe}_{2}\right)_{2}\right\}\left(\kappa^{3}\right.\right.$-bpzbdeape $\left.)\right](8)$ in $\mathrm{C}_{6} \mathrm{D}_{6}$.
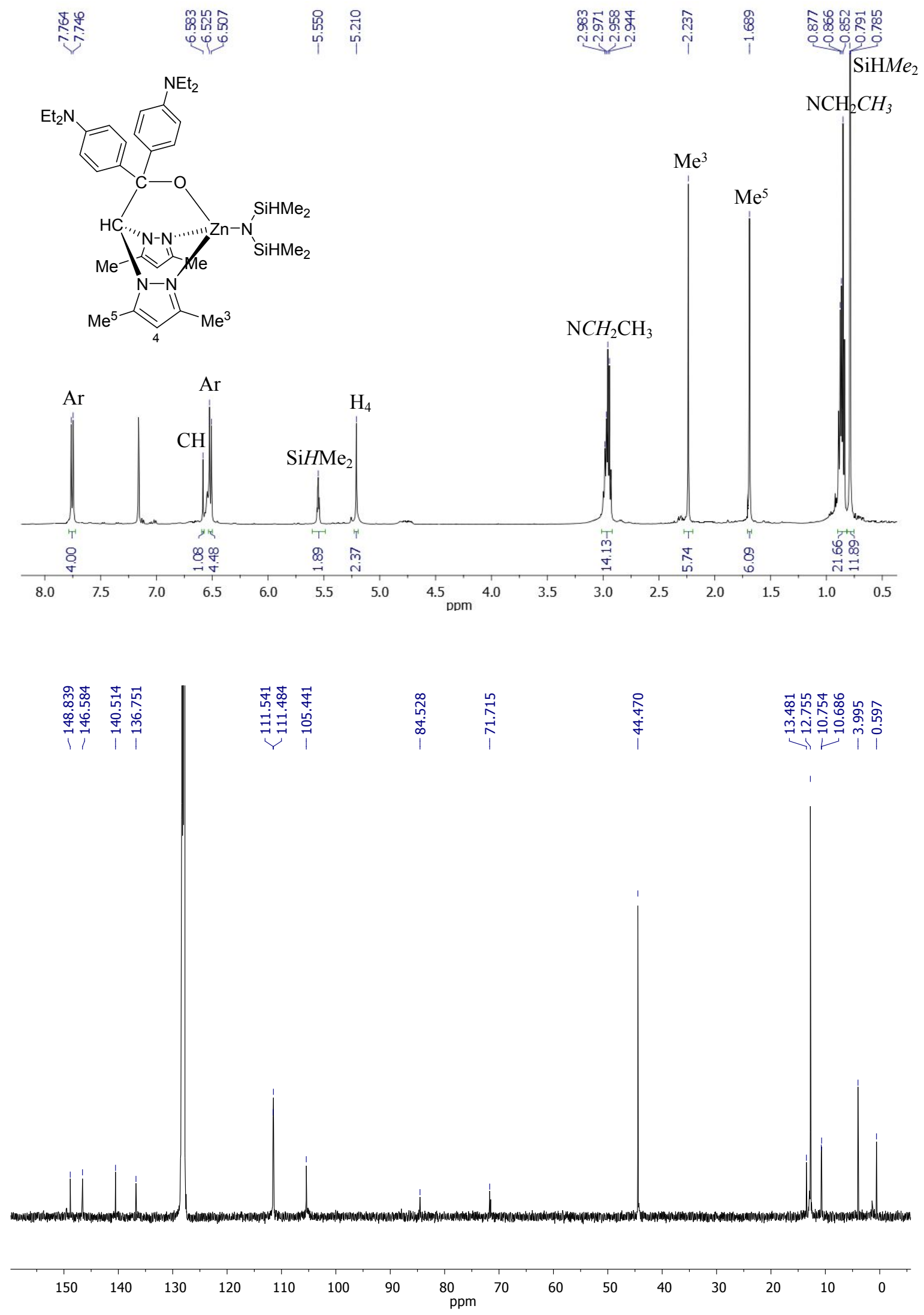
Figure S9. $\mathrm{NMR}$ spectra for $\left[\mathrm{Zn}\left\{\mathrm{C} \equiv \mathrm{C}\left(\mathrm{C}_{6} \mathrm{H}_{4}\right) \mathrm{CH}_{2} \mathrm{OH}\right\}\left(\kappa^{3}\right.\right.$-bpzbdmape $\left.)\right](15)$ in $\mathrm{CD}_{2} \mathrm{Cl}_{2}$.
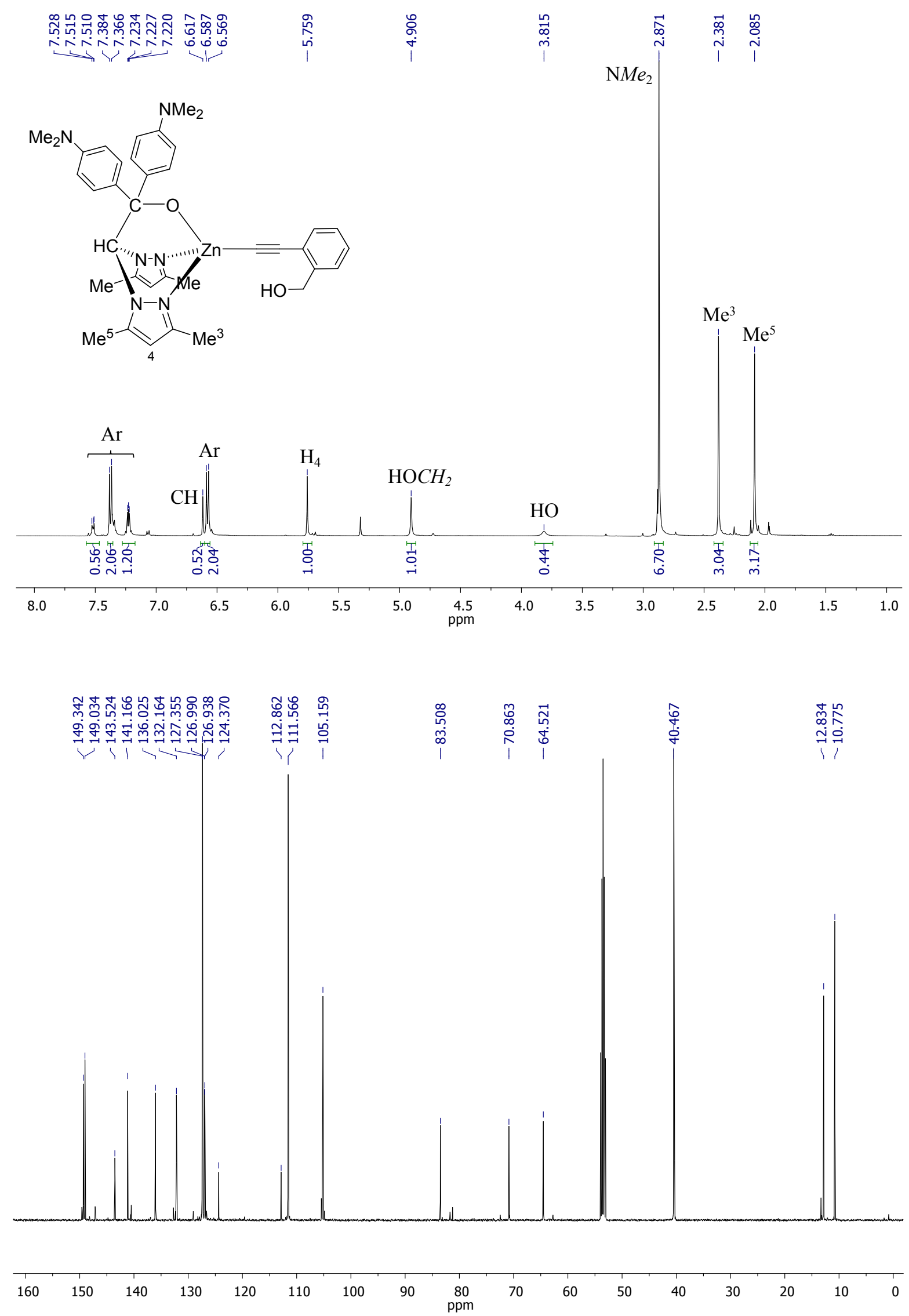
Figure S9. ${ }^{1} \mathrm{H}$ NMR monitoring of the hydroalkoxylation of 9 to 10 mediated by catalyst 8 in toluene$d^{8}$ at $90^{\circ} \mathrm{C}$.<smiles>C#Cc1ccccc1CO</smiles>

9

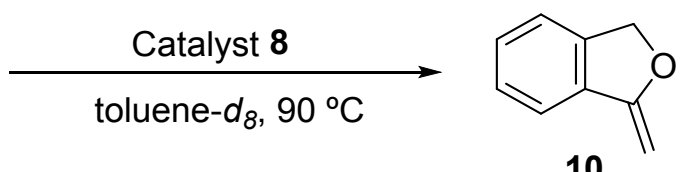

10

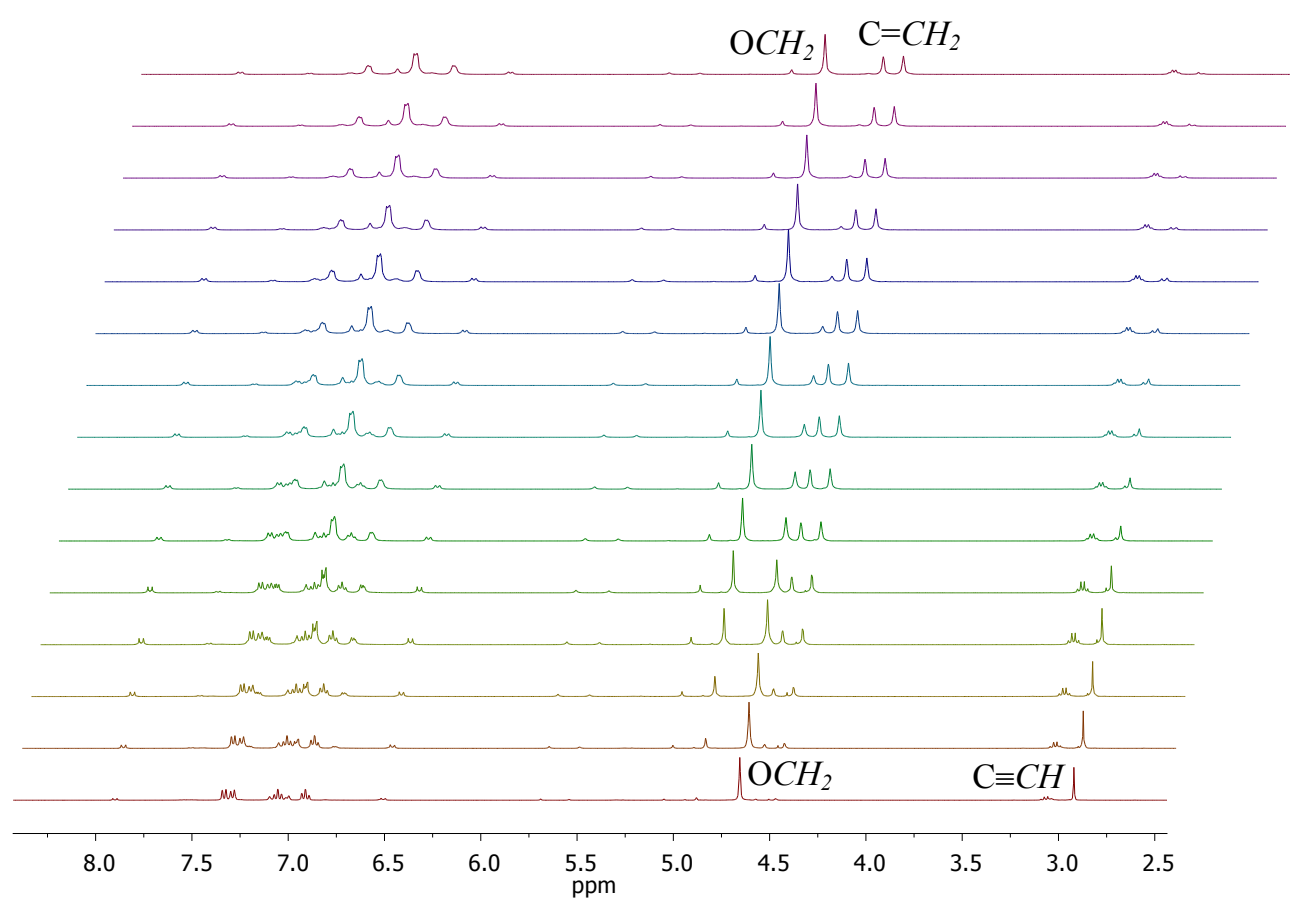

Figure S10. Plot of [9] versus reaction time for the hydroalkoxylation of $\mathbf{9}$ catalyzed by catalyst 8 at different concentrations of $\mathbf{8}$. Conditions: $[\mathbf{9}]=0.55 \mathrm{M},[8]=0.0070-0.0353 \mathrm{M}$.

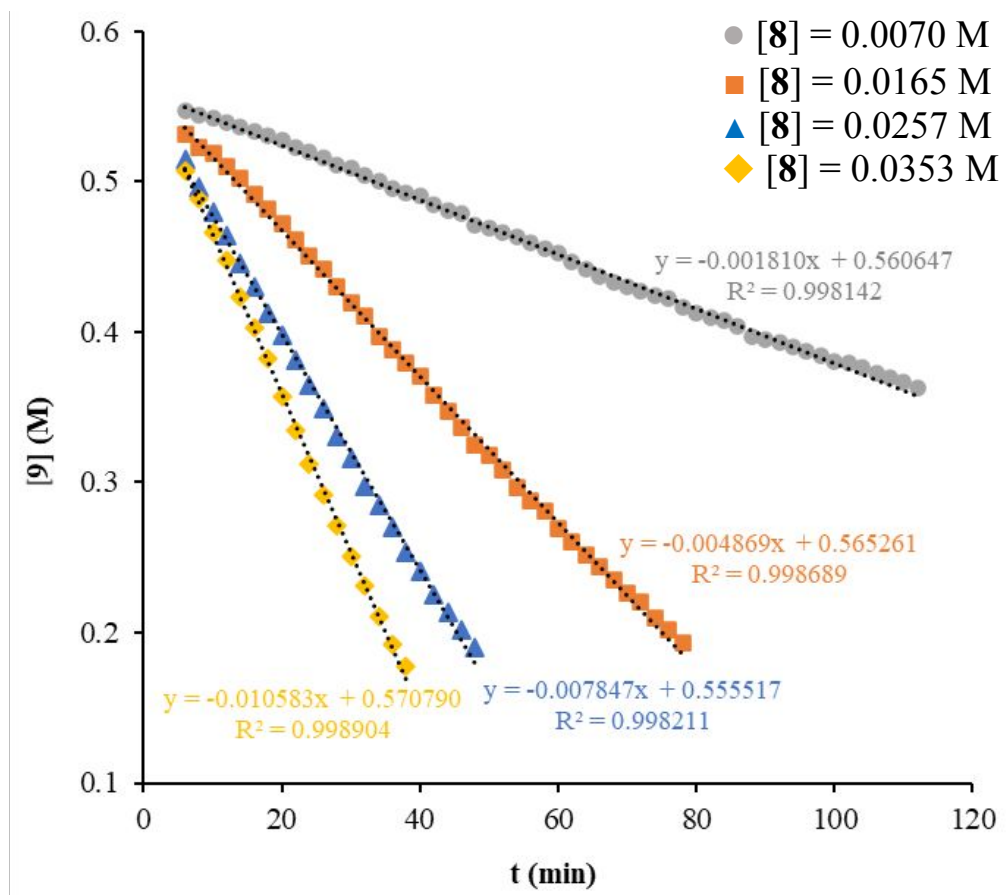


Figure S11. Arrhenius plot for the cyclization of 9 catalyzed by 8 over the temperature range 60-90 ${ }^{\circ} \mathrm{C}$ in toluene- $d^{8}$.

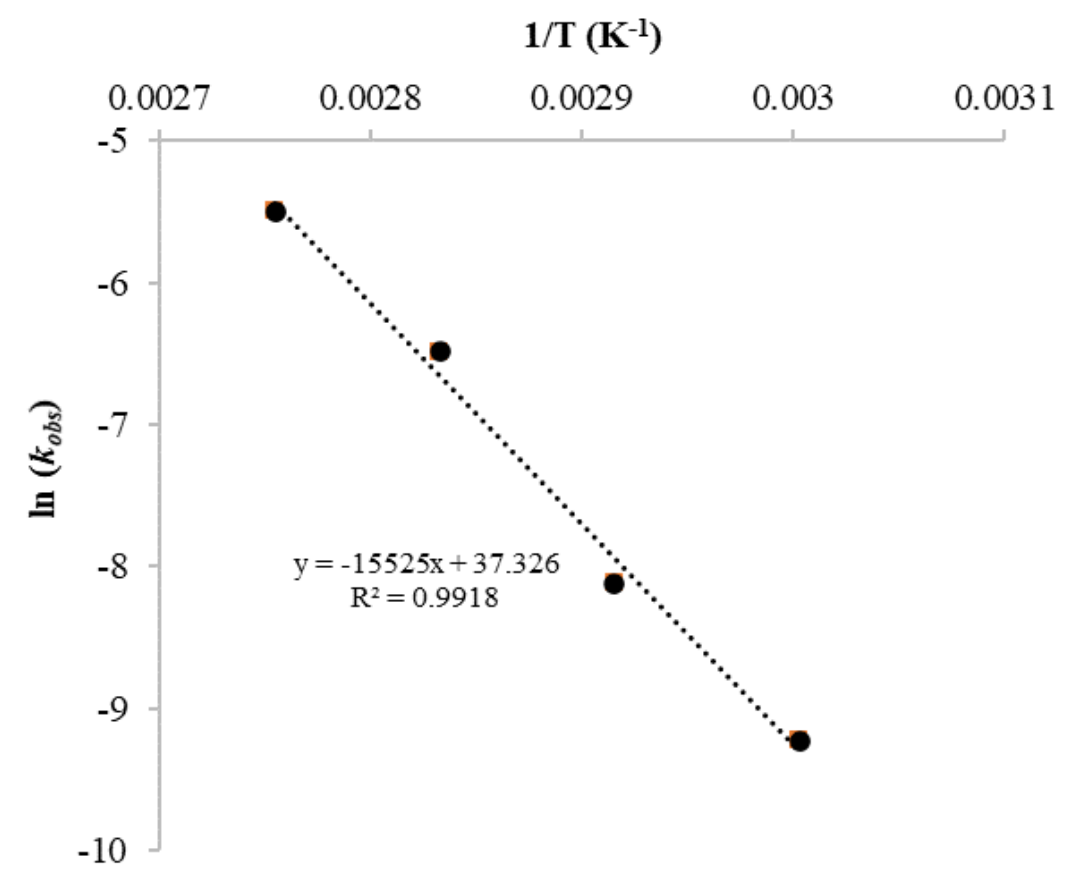

Figure S12. Eyring plot for the cyclization of 9 catalyzed by 8 over the temperature range $60-90{ }^{\circ} \mathrm{C}$ in toluene- $d^{8}$.

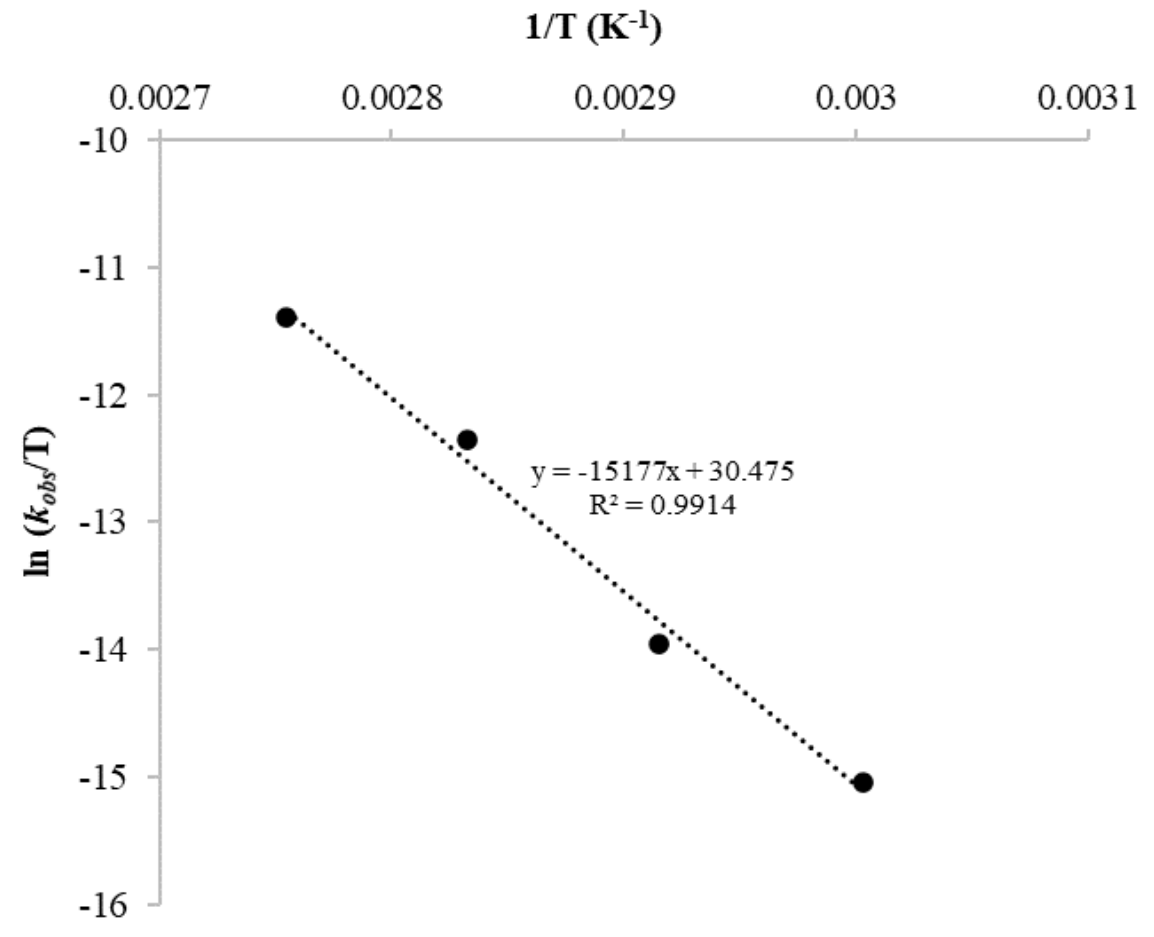


Table S1. Crystal data and structure refinement for compounds $2 \times 1.25 C_{7} H_{8}, 3 \times C_{7} H_{8}$ and $15 \times C D C l_{3}$.

\begin{tabular}{|c|c|c|c|}
\hline & $2 \times 1.25 \mathrm{C}_{7} \mathrm{H}_{8}$ & $3 \mathrm{xC}_{7} \mathrm{H}_{8}$ & $15 \times \mathrm{CDCl}_{3}$ \\
\hline Empirical formula & $\begin{array}{c}\mathrm{C}_{30} \mathrm{H}_{40} \mathrm{~N}_{6} \mathrm{OZn} \\
\cdot 1.25\left(\mathrm{C}_{7} \mathrm{H}_{8}\right) \\
\end{array}$ & $\begin{array}{c}\mathrm{C}_{41} \mathrm{H}_{61} \mathrm{~N}_{7} \mathrm{OSi}_{2} \mathrm{Zn} \\
\cdot\left(\mathrm{C}_{7} \mathrm{H}_{8}\right)\end{array}$ & $\begin{array}{c}\mathrm{C}_{38} \mathrm{H}_{43} \mathrm{Cl}_{3} \mathrm{~N}_{6} \mathrm{O}_{2} \mathrm{Zn} \\
\cdot\left(\mathrm{CDCl}_{3}\right)\end{array}$ \\
\hline Formula weight & 681.21 & 789.51 & 787.50 \\
\hline Temperature $(\mathrm{K})$ & $230(2)$ & $110(2)$ & $240(2)$ \\
\hline Wavelength $(\AA)$ & 0.71073 & 0.71073 & 0.71073 \\
\hline Crystal system & Triclinic & Triclinic & Triclinic \\
\hline Space group & $\bar{P} \overline{1}$ & $P \overline{1}$ & $\mathrm{P} \overline{1}$ \\
\hline $\mathrm{a}(\AA)$ & $8.280(4)$ & $8.7835(8)$ & $9.049(3)$ \\
\hline $\mathrm{b}(\AA)$ & $13.612(7)$ & $16.0259(16)$ & $12.419(4)$ \\
\hline$c(\AA)$ & $18.807(9)$ & $16.5683(16)$ & $17.602(5)$ \\
\hline$\alpha\left(^{\circ}\right)$ & $94.659(7)$ & $113.168(3)$ & $97.526(3)$ \\
\hline$\beta\left(^{\circ}\right)$ & $90.141(6)$ & $90.200(3)$ & $95.021(3)$ \\
\hline$\gamma\left({ }^{\circ}\right)$ & $95.510(7)$ & $93.545(3)$ & $95.922(3)$ \\
\hline Volume $\left(\AA^{3}\right)$ & $2102.8(18)$ & $2139.0(4)$ & $1940.4(10)$ \\
\hline $\mathrm{Z}$ & 2 & 2 & 2 \\
\hline $\begin{array}{c}\text { Density (calculated) } \\
\left(\mathrm{g} / \mathrm{cm}^{3}\right)\end{array}$ & 1.076 & 1.226 & 1.348 \\
\hline $\begin{array}{l}\text { Absorption coefficient } \\
\qquad\left(\mathrm{mm}^{-1}\right)\end{array}$ & 0.617 & 0.669 & 0.880 \\
\hline $\mathrm{F}(000)$ & 725 & 844 & 820 \\
\hline Crystal size $\left(\mathrm{mm}^{3}\right)$ & $0.32 \times 0.24 \times 0.14$ & $0.19 \times 0.15 \times 0.08$ & $0.32 \times 0.25 \times 0.16$ \\
\hline Index ranges & $\begin{array}{l}-10 \leq \mathrm{h} \leq 10 \\
-15 \leq \mathrm{k} \leq 17 \\
-23 \leq 1 \leq 23\end{array}$ & $\begin{aligned}-10 & \leq \mathrm{h} \leq 10 \\
-20 & \leq \mathrm{k} \leq 20 \\
-20 & \leq 1 \leq 20\end{aligned}$ & $\begin{array}{r}-10 \leq \mathrm{h} \leq 10 \\
-14 \leq \mathrm{k} \leq 13 \\
-20 \leq 1 \leq 20\end{array}$ \\
\hline Reflections collected & 14361 & 99593 & 11202 \\
\hline Independent reflections & $\begin{array}{c}8241 \\
{[\mathrm{R}(\text { int })=0.2107]}\end{array}$ & $\begin{array}{c}8719 \\
{[\mathrm{R}(\mathrm{int})=0.1231]}\end{array}$ & $\begin{array}{c}6418 \\
{[\mathrm{R}(\mathrm{int})=0.0604]}\end{array}$ \\
\hline $\begin{array}{c}\text { Data / restraints / } \\
\text { parameters }\end{array}$ & $8241 / 216 / 501$ & 8719 / $105 / 535$ & $6418 / 0 / 460$ \\
\hline Goodness-of-fit on $\mathrm{F}^{2}$ & 0.805 & 1.021 & 1.085 \\
\hline Final $\mathrm{R}$ indices $[I>2 \sigma(I)]$ & $\begin{array}{l}\mathrm{R} 1=0.0805, \\
\mathrm{wR} 2=0.1924\end{array}$ & $\begin{array}{c}\mathrm{R} 1=0.0458, \\
\mathrm{wR} 2=0.0938\end{array}$ & $\begin{array}{l}\mathrm{R} 1=0.0813, \\
\mathrm{wR} 2=0.1475\end{array}$ \\
\hline $\begin{array}{c}\text { Largest diff. peak / hole, } \\
\text { e. } \AA^{-3}\end{array}$ & 0.506 and -0.529 & 0.766 and -0.553 & 0.318 and -0.455 \\
\hline
\end{tabular}


Table S2. Selected bond distances $(\AA)$ and angles $\left(^{\circ}\right)$ for compounds $2 \times 1.25 C_{7} \mathbf{H}_{8}, 3 \times C_{7} \mathbf{H}_{8}$ and $15 \times \mathrm{CDCl}_{3}$.

\begin{tabular}{|c|c|c|c|}
\hline \multicolumn{4}{|c|}{$2 \times 1.25 \mathrm{C}_{7} \mathrm{H}_{8}$} \\
\hline \multicolumn{2}{|c|}{ Bond distances $(\AA)$} & \multicolumn{2}{|c|}{ Angles $\left(^{\circ}\right)$} \\
\hline $\mathrm{Zn}(1)-\mathrm{O}(1)$ & $1.905(4)$ & $\mathrm{O}(1)-\mathrm{Zn}(1)-\mathrm{N}(1)$ & $92.69(16)$ \\
\hline $\mathrm{Zn}(1)-\mathrm{N}(1)$ & $2.105(5)$ & $\mathrm{O}(1)-\mathrm{Zn}(1)-\mathrm{N}(3)$ & $89.09(16)$ \\
\hline $\mathrm{Zn}(1)-\mathrm{N}(3)$ & $2.159(5)$ & $\mathrm{N}(1)-\mathrm{Zn}(1)-\mathrm{N}(3)$ & $84.54(17)$ \\
\hline $\mathrm{Zn}(1)-\mathrm{C}(29)$ & $1.957(7)$ & $\mathrm{N}(1)-\mathrm{Zn}(1)-\mathrm{C}(29)$ & $121.0(3)$ \\
\hline $\mathrm{C}(29)-\mathrm{C}(30)$ & $1.442(13)$ & $\mathrm{O}(1)-\mathrm{Zn}(1)-\mathrm{C}(29)$ & $134.4(3)$ \\
\hline $\mathrm{O}(1)-\mathrm{C}(12)$ & $1.366(6)$ & $\mathrm{N}(3)-\mathrm{Zn}(1)-\mathrm{C}(29)$ & $121.4(3)$ \\
\hline \multicolumn{4}{|c|}{$3 \times \mathrm{C}_{7} \mathrm{H}_{8}$} \\
\hline \multicolumn{2}{|c|}{ Bond distances $(\AA ̊)$} & \multicolumn{2}{|c|}{ Angles ( $\left.{ }^{\circ}\right)$} \\
\hline $\mathrm{Zn}(1)-\mathrm{O}(1)$ & $1.9124(19)$ & $\mathrm{O}(1)-\mathrm{Zn}(1)-\mathrm{N}(1)$ & $91.97(9)$ \\
\hline $\mathrm{Zn}(1)-\mathrm{N}(1)$ & $2.086(2)$ & $\mathrm{O}(1)-\mathrm{Zn}(1)-\mathrm{N}(3)$ & $88.66(9)$ \\
\hline $\mathrm{Zn}(1)-\mathrm{N}(3)$ & $2.091(2)$ & $\mathrm{N}(1)-\mathrm{Zn}(1)-\mathrm{N}(3)$ & $89.72(9)$ \\
\hline $\mathrm{Zn}(1)-\mathrm{N}(7)$ & $1.904(2)$ & $\mathrm{O}(1)-\mathrm{Zn}(1)-\mathrm{N}(7)$ & $125.35(9)$ \\
\hline $\mathrm{O}(1)-\mathrm{C}(12)$ & $1.374(3)$ & $\mathrm{N}(7)-\mathrm{Zn}(1)-\mathrm{N}(1)$ & $126.92(10)$ \\
\hline $\operatorname{Si}(1)-N(7)$ & $1.714(2)$ & $\mathrm{N}(7)-\mathrm{Zn}(1)-\mathrm{N}(3)$ & $123.08(10)$ \\
\hline $\mathrm{Si}(2)-\mathrm{N}(7)$ & $1.701(2)$ & $\mathrm{C}(12)-\mathrm{O}(1)-\mathrm{Zn}(1)$ & $120.52(16)$ \\
\hline \multicolumn{4}{|c|}{$15 \times \mathrm{CDCl}_{3}$} \\
\hline \multicolumn{2}{|c|}{ Bond distances $(\AA ̊)$} & \multicolumn{2}{|c|}{ Angles $\left(^{\circ}\right)$} \\
\hline $\mathrm{Zn}(1)-\mathrm{O}(1)$ & $1.923(3)$ & $\mathrm{O}(1)-\mathrm{Zn}(1)-\mathrm{N}(1)$ & $91.06(16)$ \\
\hline $\mathrm{Zn}(1)-\mathrm{N}(1)$ & $2.052(4)$ & $\mathrm{O}(1)-\mathrm{Zn}(1)-\mathrm{N}(3)$ & $90.26(17)$ \\
\hline $\mathrm{Zn}(1)-\mathrm{N}(3)$ & $2.071(5)$ & $\mathrm{N}(1)-\mathrm{Zn}(1)-\mathrm{N}(3)$ & $89.43(18)$ \\
\hline $\mathrm{Zn}(1)-\mathrm{C}(29)$ & $1.926(6)$ & $\mathrm{O}(1)-\mathrm{Zn}(1)-\mathrm{C}(29)$ & $133.7(2)$ \\
\hline $\mathrm{O}(1)-\mathrm{C}(12)$ & $1.396(6)$ & $\mathrm{C}(29)-\mathrm{Zn}(1)-\mathrm{N}(1)$ & $122.2(2)$ \\
\hline $\mathrm{O}(2)-\mathrm{C}(37)$ & $1.390(7)$ & $\mathrm{C}(29)-\mathrm{Zn}(1)-\mathrm{N}(3)$ & $118.4(2)$ \\
\hline $\mathrm{C}(11)-\mathrm{C}(12)$ & $1.586(7)$ & $\mathrm{C}(12)-\mathrm{O}(1)-\mathrm{Zn}(1)$ & $120.0(3)$ \\
\hline
\end{tabular}




\section{References}

(1) Seo, S. Y.; Yu, X.; Marks, T. J. Intramolecular Hydroalkoxylation/Cyclization of Alkynyl Alcohols Mediated by Lanthanide Catalysts. Scope and Reaction Mechanism. J. Am. Chem. Soc. 2009, 131, 263-276.

(2) Pale, P.; Chuche, J. Silver-Catalyzed Cyclization of Acetylenic Alcohols: Synthesis of Functionalized 2-Methylene-oxolanes. Eur. J. Org. Chem. 2000, 1019-1025. 